\title{
A NEW PROOF OF THE REIDEMEISTER- SINGER THEOREM ON STABLE EQUIVALENCE OF HEEGAARD SPLITTINGS
}

\author{
ROBERT CRAGGS
}

\begin{abstract}
A proof of the Reidemeister-Singer theorem on stable equivalence of Heegaard splittings is given. This proof makes use of the Chillingworth theorem on the preservation of simplicial collapses for subdivisions of complexes of dimension less than or equal to three, and it is based on the observation that subdivision and collapsing preserve stable equivalence.
\end{abstract}

The Reidemeister-Singer theorem on Heegaard splittings of 3-manifolds [Re], [Si] provides that any two Heegaard splittings of a 3-manifold are stably equivalent. Although this theorem has been around since the thirties, probably the most significant application of it thus far did not come until the late sixties when Waldhausen [Wd] proved that all Heegaard splittings of the 3 -sphere are standard. Unfortunately the proofs in both [Re] and [Si] appear to be obscure at the same point: they seem to anticipate, by about thirty years, Chillingworth's theorem [Ch] on the invariance of simplicial collapses under subdivision for rectilinear simplicial complexes of dimension less than or equal to three. Both Papakyriakopoulos and Waldhausen suggested to us several years ago that a complete proof of the stable equivalence theorem ought to appear somewhere. We obtained the proof given here after Joe Martin told us that the Topology Seminar at The University of Wisconsin had used the Chillingworth theorem to reconstruct a proof of the Reidemeister-Singer theorem.

The basic idea of the proof is that subdivision and simplicial collapsing preserve stable equivalence. In another paper (see $[\mathbf{C r}]$ ) we use some fairly intricate refinements of this idea to show that simply connected 4-dimensional cobordisms between connected 3-manifolds are stably classified, that is, up to connected sum with copies $S^{2} \times S^{2}$, by certain Heegaard representations for 3-manifolds.

Everything here is either in the pwl- or the simplicial category. Thus, in particular, maps and homeomorphisms are always pwl or simplicial. A complex is a finite rectilinear simplicial complex in some Euclidean space $E^{n}$. A polyhedron is the carrier of a complex, and for a complex $K$ we denote this carrier by $|K|$. A triangulation of a polyhedron $P$ is a complex $K$ such that $|K|=P$. If $K$ is a complex and some subcomplex either triangulates a

Received by the editors March 27, 1974 and, in revised form, August 26, 1975.

AMS (MOS) subject classifications (1970). Primary 57Cxx, 57A10.

Key words and phrases. Heegaard splitting, stable equivalence, collapse, subdivision.

- American Mathematical Society 1976 
polyhedron $P$ or subdivides a complex $L$, then we denote this subcomplex by $K(P)$ or $K(L)$. If $L$ is a subcomplex of a complex $K$ and $K^{\prime}$ subdivides $K$, then we denote by $L^{\prime}$ the induced subdivision of $L$. For a complex $K$, we denote the $i$-skeleton of $K$ by $K_{i}$. For a simplex $\tau$, we denote by $[\tau]$ the smallest complex containing $\tau$. For a subcomplex $L$ of a complex $K$, we denote by $S(L, K)$ the simplicial neighborhood of $L$ in $K$, that is, the smallest subcomplex of $K$ whose carrier contains a neighborhood of $|L|$. A graph is a connected 1complex.

An n-manifold is a polyhedron such that each point has a neighborhood which is an $n$-ball. Unless we specify to the contrary, it is assumed that a manifold has no boundary. For an oriented manifold $M$, we denote by $-M$ the same manifold with the opposite orientation. Let $B^{4}$ denote the 4-ball $[-1,1]^{4} \subseteq E^{4}$ and let $\Sigma$ denote the boundary of $B^{4}$. We assume that $\Sigma$ is provided with a fixed but unspecified orientation. Let $G$ be a graph and $M$ a 3-manifold which contains $|G|$. We say that $|G|$ is admissible in $M$ provided that for some regular neighborhood $U$ of $|G|$ in $M$, the complementary manifold $V=M \backslash$ Int $U$ is a cube-with-handles.

Let $M$ and $\tilde{M}$ be oriented $n$-manifolds. We form the connected sum $M \# \tilde{M}$ as follows: Locate $n$-balls $B$ and $\tilde{B}$ in Int $M$ and Int $\tilde{M}$. Let $g: \operatorname{Bd} B \rightarrow \operatorname{Bd} \tilde{B}$ be an orientation reversing homeomorphism. Form the identification space $(M \backslash \operatorname{Int} B)+{ }_{g}(\tilde{M} \backslash \operatorname{Int} \tilde{B})$. For $M \# \tilde{M}$ take some polyhedron $\tilde{\tilde{M}}$ which is homeomorphic to this identification space under an orientation preserving homeomorphism. For manifolds which have boundary, it is also convenient to have a boundary sum $M \#_{b} \tilde{M}$. This is formed by choosing $n$-balls $B$ and $\tilde{B}$ in $M$ and $\tilde{M}$ so that $B \cap \operatorname{Bd} M$ and $\tilde{B} \cap \operatorname{Bd} \tilde{M}$ are $(n-1)$-balls. Then an orientation reversing homeomorphism $g: \operatorname{Fr} B \rightarrow \operatorname{Fr} \tilde{B}$ is chosen and the identification space $\mathrm{Cl}(\boldsymbol{M} \backslash B)+{ }_{g} \mathrm{Cl}(\tilde{M} \backslash \tilde{B})$ is formed. The boundary sum $M \sharp_{b} \tilde{M}$ is taken to be some polyhedron $\tilde{\tilde{M}}$ which is homeomorphic to the identification space under an orientation preserving homeomorphism. It is easy to verify that these sums are associative and commutative.

A Heegaard splitting of genus $n$ of an oriented 3-manifold $M$ is a triple $(M, U, V)$ such that (1) $U$ and $V$ are oriented cubes-with-handles of genus $n$ in $M$ which receive their orientations from their inclusions in $M$, (2) the union of $U$ and $V$ is $M$, and (3) $U$ and $V$ share the same boundary and have disjoint interiors. Some people call a Heegaard splitting of $M$ a pair $(M, S)$ where $S$ is an oriented surface which splits $M$ into a pair of cubes-with-handles. Under this description $(M, U, V)$ would become $(M, \mathrm{Bd} U)$ and $(M, V, U)$ would become $(M, \mathrm{Bd} V)=(M,-\mathrm{Bd} U)$. Two Heegaard splittings $(M, U, V)$ and $(\tilde{M}, \tilde{U}, \tilde{V})$ are equivalent if there is an orientation preserving homeomorphism $h$ which takes the triple $(M, U, V)$ to the triple $(\tilde{M}, \tilde{U}, \tilde{V})$.

The connected sum $(M, U, V) \#(\tilde{M}, \tilde{U}, \tilde{V})$ of Heegaard splittings $(M, U, V)$ and $(\tilde{M}, \tilde{U}, \tilde{V})$ is formed as follows: First $\tilde{M}=M \# \tilde{M}$ is formed by choosing the 3-balls $B$ and $\tilde{B}$ and the homeomorphism $g$ so that $B \cap \mathrm{Bd} U$ and $\tilde{B} \cap \mathrm{Bd} \tilde{U}$ are spanning disks in $B$ and $\tilde{B}$ and so that $g(U \cap \tilde{\tilde{V}} \operatorname{Bd} B)$ $=\tilde{U} \cap \operatorname{Bd} \tilde{B}$. Then there are subspaces $\tilde{\tilde{U}}$ and $\tilde{\tilde{V}}$ of $\tilde{\tilde{M}}$ such that $\tilde{\tilde{U}}$ and $\tilde{V}$ correspond to the boundary sums $U \sharp_{b} \tilde{U}$ and $V \sharp_{b} \tilde{V}$, and we take $(\tilde{\tilde{M}}, \tilde{\tilde{U}}, \tilde{V})$ to be the connected sum $(M, U, V) \#(\tilde{M}, \tilde{U}, \tilde{V})$. It is clear as before that connected sum is well defined up to equivalence and that it is associative and 
commutative. Let $\eta$ denote the Heegaard splitting of genus 1 of $\Sigma$ (there is only one up to equivalence). Let $k \eta$ denote the $k$-fold connected sum of $\eta$. We say that two Heegaard splittings $(M, U, V)$ and $(\tilde{M}, \tilde{U}, \tilde{V})$ are stably equivalent provided that there are integers $p$ and $\tilde{p}$ such that $(M, U, V) \# p \eta$ and $(\tilde{M}, \tilde{U}$, $\tilde{V}) \# \tilde{p} \eta$ are equivalent.

Lemma 1. Suppose that $J$ is a finite complex of dimension at most 3, suppose that $K$ is a subcomplex of $J_{2}$ with $K_{1}=J_{1}$, and suppose that $J^{\prime}$ is a subdivision of $J$.

Then there is a subcomplex $L$ of $\left(J^{\prime}\right)_{2}$ such that $L_{1}=\left(J^{\prime}\right)_{1}$ and $L \bigvee^{s} K^{\prime}$.

Proof. Let $\tau_{1}, \ldots, \tau_{i}, \ldots, \tau_{m}$ denote the 3 -simplexes of $J$, and let $\Delta_{1}, \ldots$, $\Delta_{j}, \ldots, \Delta_{n}$ denote the 2-simplexes of $J \backslash K$. From [Ch] we know that in each $\left(\dot{\tau}_{i}\right)^{\prime}$ there is a 2-simplex $\gamma_{i}$ such that $\left[\tau_{i}\right]^{\prime} V^{s}\left(\dot{\tau}_{i}\right)^{\prime} \backslash \gamma_{i}$. These collapses can be carried out in order of decreasing dimension [Ze, Lemma 11]; thus there is a 2-subcomplex $L\left(\tau_{i}\right)$ of each $\left[\tau_{i}\right]^{\prime}$ such that $L\left(\tau_{i}\right)$ contains no 2-simplex of $\left(\dot{\tau}_{i}\right)^{\prime}$, the complex $\left(\left[\tau_{i}\right]^{\prime}\right)_{1}$ is a subcomplex of $L\left(\tau_{i}\right)$, and $L\left(\tau_{i}\right) v^{s}\left(\left(\tau_{i}\right)^{\prime}\right)_{1}$. For each 2simplex $\Delta_{i}$ let $L\left(\Delta_{i}\right)$ be formed from $\left[\Delta_{i}\right]^{\prime}$ by removing any 2 -simplex. Then $L\left(\Delta_{i}\right) v^{s}\left(\dot{\Delta}_{i}\right)^{\prime}$.

For $0 \leqslant i \leqslant m$, set

$$
L(i)=K^{\prime} \cup \cup\left\{L\left(\tau_{i}\right): j>i\right\} \cup \cup\left\{L\left(\Delta_{i}\right): i \leqslant n\right\},
$$

and for $m+1 \leqslant i \leqslant m+n$, set

$$
L(i)=K^{\prime} \cup \cup\left\{L\left(\Delta_{j}\right): j>i-m\right\} .
$$

Then we have

$$
L=L(0) \searrow^{s} L(1) \searrow^{s} \ldots \searrow^{s} L(m+n)=K^{\prime} .
$$

LEMMA 2. Suppose that $G$ is a graph and $M$ is a 3-manifold such that $|G|$ is admissible in $M$.

Then there is a triangulation $T$ of $M$ and there is a subcomplex $T(1)$ of $T_{2}$ such that $T(1)_{1}=T_{1}$ and $T(1) \vee^{s} G^{\prime}$ where $G^{\prime}$ is a subdivision of $G$.

Proof. First suppose that there is a complex $J$ such that (1) $|J|$ is homeomorphic to $M$ under a homeomorphism $h:|J| \rightarrow M$, (2) there is a subcomplex $H$ of $J$ such that $h(|H|)=|G|$, and (3) the 2-skeleton $J_{2}$ contains a subcomplex $K$ such that $K_{1}=J_{1}$ and $K \vee^{s} H$. Then there is a triangulation $T$ of $M$ containing a subcomplex $G^{\prime}$ that subdivides $G$, and there is a subdivision $J^{\prime}$ of $J$ such that $h: J^{\prime} \rightarrow T$ is a simplicial isomorphism. But $K^{\prime} \vee^{\prime} H^{\prime}$ by [Ch], and by Lemma 1 there is a subcomplex $L$ of $\left(J^{\prime}\right)_{2}$ such that $L_{1}=\left(J^{\prime}\right)_{1}$ and $L V^{s} K^{\prime} V^{s} H^{\prime}$. For $T(1)$ we take $h(L)$ and we use the isomorphism $h$ to obtain the collapse $T(1) \bigvee^{s} G^{\prime}$.

The proof of the lemma reduces then to a proof that there is a complex $J$ which satisfies the three conditions in the preceding paragraph.

Let $T(2)$ be a triangulation of $M$ which contains a subcomplex $G(1)$ subdividing $G$. Let $H$ denote the second barycentric subdivision $G(1)^{2}$ and consider the simplicial neighborhood $N(1)=S\left(H, T(2)^{2}\right)$ where $T(2)^{2}$ denotes the second barycentric subdivision of $T(2)$. Now $N(1) \searrow^{s} H$, and since 
$|G|$ is admissible in $M$ and $|N(1)|$ is a regular neighborhood of $|G|$, both $N(1)$ and $N(2)=T(2)^{2} \backslash \stackrel{\circ}{N}(1)$ are cubes-with-handles of genus say $n$. The handlebody $|N(2)|$ is a union of 3-cells $\cup\left\{C_{i}: 0 \leqslant i \leqslant n\right\}$ where $C_{i} \cap C_{j}$ $=\varnothing$ for $i \neq j$ and $i \neq 0$ and $j \neq 0$, and $C_{i} \cap C_{0}$ is a union of two disjoint disks $D_{i}$ and $E_{i}$ for $i \neq 0$. Furthermore, if $n \neq 0$ then $|N(2)|$ has a spine $Y$ in its interior such that (1) $Y$ is a bouquet of $n$ circles, (2) each $Y \cap C_{i}(i \geqslant 1)$ is an unknotted spanning arc, and (3) $Y \cap C_{0}$ is an unknotted cone with cone point $y$ where all of the noncutpoints of $Y \cap C_{0}$ lie in $\mathrm{Bd} C_{0}$. If $n=1$, specify any point of $Y \cap$ Int $C_{0}$ as the cone point $y$, and if $n=0$ take a point in Int $C_{0}$ to be $Y=y$. Set $x_{i}=Y \cap D_{i}$ and $z_{i}=Y \cap E_{i}$. Let $(\dot{N}(1))^{\prime}$ be a subdivision of $\dot{N}(1)$ which contains a subcomplex triangulating $\left(\cup \operatorname{Bd} D_{i}\right)$ $\cup\left(\cup \operatorname{Bd} E_{i}\right)$. Extend $(\dot{N}(1))^{\prime}$ to a subdivision $N(1)^{\prime}$ of $N(1)$.

In some high dimensional Euclidean space choose points $u_{0}, u_{1}, \ldots, u_{n}, v_{1}$, $\ldots, v_{n}, w_{1}, \ldots, w_{n}$ which are vertices of a simplex $\sigma$ such that $[\sigma]$ is joinable to $N(1)^{\prime}$. Form in order the following complexes:

(1) $J\left(D_{i}\right)=v_{i} * N(1)^{\prime}\left(\mathrm{Bd} D_{i}\right), i=1,2, \ldots$,

(2) $J\left(E_{i}\right)=w_{i} * N(1)^{\prime}\left(\mathrm{Bd} E_{i}\right), i=1,2, \ldots$,

(3) $J\left(C_{0}\right)=u_{0} *\left(N(1)^{\prime}\left(C_{0} \cap|N(1)|\right) \cup \cup J\left(D_{i}\right) \cup \cup J\left(E_{i}\right)\right)$, and

(4) $J\left(C_{i}\right)=u_{i} *\left(N(1)^{\prime}\left(C_{i} \cap|N(1)|\right) \cup J\left(D_{i}\right) \cup J\left(E_{i}\right)\right)$. Let $J$ denote the complex $N(1)^{\prime} \cup \cup J\left(C_{i}\right)$.

Clearly there is a homeomorphism $h:|J| \rightarrow M$ which leaves each point of $|N(1)|$ fixed. We show that there is a subcomplex $K$ of $J$ which satisfies condition (3) in the first paragraph of the proof. The complex $N(1)^{\prime}$ collapses simplicially to $H$ by [Ch]. By reordering the collapses in order of decreasing dimension (see [Ze, Lemma 11]), we find that $N(1)^{\prime} \backslash^{s} K(2) \searrow^{s} H$ where $K(2)$ is a subcomplex of $\left(N(1)^{\prime}\right)_{2}$ and $K(2)_{2}=\left(N(1)^{\prime}\right)_{1}$. Delete one 2-simplex from each $J\left(D_{i}\right)$ and each $J\left(E_{i}\right)$ and add the residual complexes to $K(2)$ to get a bigger complex $K(1)$ such that $K(1) \searrow^{s} K(2)$. Then for each $J\left(C_{i}\right)$ choose a maximal tree $X\left(C_{i}\right)$ in $\dot{J}\left(C_{i}\right)$. Add the complexes $u_{i} * X\left(C_{i}\right)$ to $K(1)$ to get a complex $K(0)$. Since each $X\left(C_{i}\right)$ contains all the vertices of $\dot{J}\left(C_{i}\right)$ we find that $K(0)_{1}=J_{1}$. Furthermore, each $X\left(C_{i}\right)$ collapses simplically to a vertex so each $u_{i} * X\left(C_{i}\right)$ collapses simplicially to the join of $u_{i}$ with a vertex and this in turn collapses simplicially to the vertex; thus these collapses induce a collapse $K(0) \searrow^{s} K(1)$. We now set $K=K(0)$ to get the simplicial collapses $K=K(0)$ $\bigvee^{s} K(1) \bigvee^{s} K(2) \searrow^{s} H$. Thus $K$ satisfies condition (3) and the proof of the lemma is complete.

Lemma 3. Suppose that $M$ is a 3-manifold and $K$ and $L$ are complexes whose carriers are in $M$. Suppose that $K \vee^{s} L$.

If either $\left|K_{1}\right|$ or $\left|L_{1}\right|$ is admissible in $M$, then so is the other, and furthermore if $(M, U, V)$ and $(M, \tilde{U}, \tilde{V})$ are Heegaard splittings of $M$ such that $(1)\left|L_{1}\right|$ $\subset$ Int $U,(2)\left|K_{1}\right| \subset$ Int $\tilde{U},(3) U \searrow\left|L_{1}\right|$, and (4) $\tilde{U} \searrow\left|K_{1}\right|$, then for some integer $p$ we have that $(M, U, V) \# p \eta$ and $(M, \tilde{U}, \tilde{V})$ are equivalent.

Proof. See [Re] or $[\mathbf{S i}]$ or $[\mathbf{W d}]$.

Theorem 4 (Reidemeister-Singer). Let $(M, U, V)$ and $(M, \tilde{U}, \tilde{V})$ be Heegaard splittings of a 3-manifold $M$. 
There are integers $p$ and $\tilde{p}$ such that $(M, U, V) \# p \eta$ and $(M, \tilde{U}, \tilde{V}) \# \tilde{p} \eta$ are equivalent.

Proof. Because $U$ and $\tilde{U}$ have 1-spines it follows from Lemmas 2 and 3 that there are integers $p_{1}$ and $\tilde{p}_{1}$, there are triangulations $T(1)$ and $\tilde{T}(1)$ of $M$, and there are regular neighborhoods $U_{1}$ and $\tilde{U}_{1}$ of $\left|T(1)_{1}\right|$ and $\left|\tilde{T}(1)_{1}\right|$ respectively such that if $V_{1}=M \backslash$ Int $U_{1}$ and $\tilde{V}_{1}=M \backslash$ Int $\tilde{U}_{1}$, then $(M, U, V)$ $\# p_{1} \eta$ and $(M, \tilde{U}, \tilde{V}) \# \tilde{p}_{1} \eta$ are respectively equivalent to $\left(M, U_{1}, V_{1}\right)$ and $(M$, $\left.\tilde{U}_{1}, \tilde{V}_{1}\right)$. Let $T$ be a triangulation of $M$ which subdivides both $T(1)$ and $\tilde{T}(1)$ (see for example [Ze, Corollary 2 to Lemma 4]). By Lemmas 1 and 3 there are integers $p_{2}$ and $\tilde{p}_{2}$ and there is a regular neighborhood $\tilde{U}$ of $\left|T_{1}\right|$ in $M$ such that if $\tilde{V}=M \backslash \operatorname{Int} \tilde{U}$, then $(M, \tilde{U}, \tilde{V})$ is equivalent to each of $\left(M, U_{1}, V_{1}\right)$ $\# p_{2} \eta$ and $\left(M, \tilde{U}_{1}, \tilde{V}_{1}\right) \# \tilde{p}_{2} \eta$. Thus by the transitivity of equivalence, $(M, U, V)$ $\#\left(p_{1}+p_{2}\right) \eta$ is equivalent to $(M, \tilde{U}, \tilde{V}) \#\left(\tilde{p}_{1}+\tilde{p}_{2}\right) \eta$; so we can take $p$ and $\tilde{p}$ to be $p_{1}+p_{2}$ and $\tilde{p}_{1}+\tilde{p}_{2}$.

\section{REFERENCES}

[Ch] D. R. J. Chillingworth, Collapsing three-dimensional convex polyhedra, Proc. Cambridge Philos. Soc. 63 (1967), 353-357. MR 35 \#995.

[Cr] R. Craggs, Relating representations for 3-and 4-manifolds (mimeographed manuscript).

[Re] K. Reidemeister, Zur dreidimensionalen Topologie, Abh. Math. Sem. Univ. Hamburg 9 (1933), 189-194.

[Si] J. Singer, Three dimensional manifolds and their Heegaard diagrams, Trans. Amer. Math. Soc. 35 (1933), 88-111.

[Wd] F. Waldhausen, Heegaard-Zerlegungen der 3-Sphäre, Topology 7 (1968), 195-203. MR 37 \#3576.

[Ze] E. C. Zeeman, Seminar on combinatorial topology, Publ. Hautes Études Sci., Paris, 1963 (mimeographed notes).

Department of Mathematics, University of Illinois, Urbana, Illinois 61801 